\title{
Intermédialités
}

Histoire et théorie des arts, des lettres et des techniques

Intermediality

History and Theory of the Arts, Literature and Technologies

\section{Broder les rythmes}

\section{À propos de la Tapisserie de Bayeux}

\section{Jean-Claude Schmitt}

Numéro 16, automne 2010

rythmer

rhythmize

URI : https://id.erudit.org/iderudit/1001954ar

DOI : https://doi.org/10.7202/1001954ar

Aller au sommaire du numéro

\section{Éditeur(s)}

Revue intermédialités (Presses de l’Université de Montréal)

ISSN

1705-8546 (imprimé)

1920-3136 (numérique)

Découvrir la revue

Citer cet article

Schmitt, J.-C. (2010). Broder les rythmes : à propos de la Tapisserie de Bayeux. Intermédialités / Intermediality, (16), 23-34. https://doi.org/10.7202/1001954ar
Résumé de l'article

Cette rapide étude des rythmes de la Tapisserie de Bayeux s'inscrit dans une réflexion bien plus large portant sur l'histoire des rythmes au Moyen Âge. Celle-ci concerne notamment les rythmes des images et ceux de la narration historique, ce qui justifie l'examen de la célèbre broderie. Cette étude croise l'analyse des bordures, des images de la bande centrale et des inscriptions latines. L'usage des temps verbaux suggère que l'oeuvre pouvait être commentée à haute voix lors de son exposition (attestée au $15^{\mathrm{e}}$ siècle) à l'occasion de la fête de la dédicace de la cathédrale de Bayeux. La répétition et la variété des formes, des couleurs, des sons, les accélérations et les ralentissements du mouvement des cavaliers et des fantassins, et même les temps d'arrêt et les retours en arrière, donnent à la broderie son caractère rythmique exceptionnel. 


\title{
Broder les rythmes
}

\section{$\grave{A}$ propos de la Tapisserie de Bayeux*}

\author{
Jean-Claude Schmitt
}

\section{D} epuis l'invention vers 1878, par Étienne-Jules Marey (1830-1904), de la chronophotographie ${ }^{1}$, bientôt relayée, à la veille de la Première Guerre mondiale, par le cinéma muet, puis le cinéma parlant, lui-même bousculé vers 1950 par la télévision et plus près de nous par les images numériques, «l’œuvre d'art à l'époque de sa reproductibilité technique», pour parler comme Walter Benjamin ${ }^{2}$, nous a habitués à voir les êtres et les choses se mouvoir sur nos écrans comme il nous semble les percevoir au quotidien. Pourtant, ces images mobiles, bien qu'auréolées du titre de «septième art», n’ont pas détrôné les images fixes, peintures ou photographies. Tout au plus leur ont-elles imposé leur manière de percevoir le mouvement et le temps : ainsi un «instantané » est-il une image fixe prélevée par l'objectif du photographe dans le flux du mouvement que seul le cinéma peut restituer dans la durée. L'instantané, c’est la fixité dans le mouvement. Mais l'inverse - le mouvement dans l'image fixe - existe aussi et il est probable que le développement des images mobiles en a exacerbé l'attente chez les artistes comme chez les spectateurs : en témoigne la préoccupation croissante, dans tous les courants (expressionnisme, futurisme, art abstrait, etc.) qui ont révolutionné les arts plastiques au début du $20^{\mathrm{e}}$ siècle, pour les questions liées à la «temporalité des images ${ }^{3}$ » et plus précisément au rythme: c'est par le jeu des formes et des couleurs, leur répétition et leur variation, que Paul Klee ou Roger Delaunay

* Je tiens à exprimer mes remerciements à Aurore David et Gisèle Besson pour leurs suggestions durant le séminaire que j'ai tenu à l'EHESS en 2008-2009 sur la Tapisserie de Bayeux.

1. Michel Frizot, Étienne-Jules Marey, chronophotographe, Paris, Nathan-Delpire, 2001.

2. Walter Benjamin, «L'œuvre d'art à l'époque de sa reproduction mécanisée », dans Écrits français, Paris, Gallimard, coll. «Folio. Essais», 1991, p. 177-220. L'auteur évoquait d'abord la photographie, image moderne, mais fixe.

3. Giovanni Careri, François Lissarrague, Jean-Claude Schmitt et Carlo Severi (dir.), Traditions et temporalités des images, Paris, Éditions de l'EHESS, 2009. 
animaient, faisaient vibrer et comme «chanter » leurs tableaux, qu'il leur arrivait d'intituler simplement «Rythmes», ce qui était le meilleur moyen d'exprimer la vibration communiquée par la peinture au spectateur, de désigner les effets propres de l'œuvre, plutôt que de nommer un objet et de renvoyer à l'ordre d'une «représentation »: toute la réalité de telles images tient au mouvement créé par la peinture elle-même $e^{4}$

Cependant, le mouvement n'est pas absent non plus des images figuratives plus anciennes. Souvent, il s'exprime par les gestes qui figurent la marche, le don (ou la réception) 5 . Il peut s'exprimer aussi au moyen d'images consécutives dont la proximité dans l'espace-temps du medium (par exemple un codex médiéval dont chaque page est enluminée) permet de restituer le continuum d'une narration en images, celle par exemple d'un récit biblique 6 . Il n'est pas rare non plus, au Moyen Âge, que le même personnage soit représenté deux fois dans la même image, mais dans deux positions et avec des attitudes différentes; ou encore, qu'il soit figuré une seule fois, mais en condensant deux moments différents de l'action : celui par exemple - comme à Berzé-la-Ville -, où le bourreau élève son épée pour frapper le saint et simultanément celui où le martyr ramasse déjà sa tête qui roule sur le sol ${ }^{7}$. Parfois, la taille exceptionnelle du support et la continuité de la figuration - comme sur la voûte peinte de Saint-Savin-sur-Gartempe ${ }^{8}$ ou dans certains ensembles de tapisseries destinées à parer le chœur d'une cathédrale ou les murs d'une salle d'apparat les jours de

4. Voir entre autres: Paul Klee, Das bildnerische Denken. Schriften zur Form- und Gestaltungslehre, édité par Jürg Spiller, Bâle, Benno Schwabe, 1956. Robert Delaunay, Du cubisme à l'art abstrait, documents inédits publiés par Pierre Francastel et suivis d'un catalogue de l'œuvre de Robert Delaunay par Guy Habasque, Paris, SEVPEN, 1957.

5. Michael Baxandall, L'œil du Quattrocento: l'usage de la peinture dans l'Italie de la Renaissance, trad. Yvette Delsaut, Paris, Gallimard, coll. "Bibliothèque illustrée des histoires », 1985. Mosche Barasch, Giotto and the Language of Gestures, Cambridge, Cambridge University Press, 1990. Jean-Claude Schmitt, La raison des gestes dans l'Occident médiéval, Paris, Gallimard, coll. «Bibliothèque des histoires », 1990.

6. Otto Pächt, L'enluminure médiévale: une introduction, trad. Jean Lacoste, Paris, Macula, 1997.

7. Jean-Claude Bonne, «Temporum concordia discors. Le temps dans les peintures murales romanes de Berzé-la-Ville», dans Eric Alliez, Gerhart Schröder, Barbara Cassin, Gisela Febel et Michel Narcy (dir.), Metamorphosen der Zeit, Munich, Wilhelm Fink Verlag, 1999, p. 145-175.

8. Jérôme Baschet, L’iconographie médiévale, Paris, Gallimard, coll. «Folio. Histoire », 2008. 
fête ${ }^{9}$ - offrent des conditions particulièrement favorables d'analyse des rythmes de la narration. Je ne m'attacherai ici qu'à un seul exemple bien connu, celui de la Tapisserie de Bayeux, dont les historiens n'ont pas manqué de rapprocher les procédés figuratifs de ceux du cinéma, voire de la bande dessinée ${ }^{10}$.

Ce faisant, je ne prétends pas apporter d'éléments d'interprétation bien nouveaux à l'étude d'une œuvre auscultée sans relâche et depuis longtemps par les spécialistes les plus divers. Mon intention n'est justement pas d'ajouter une étude à toutes celles qui existent déjà sur cet objet exceptionnel, mais d'insérer mes remarques, qui resteront rapides, dans un projet beaucoup plus vaste qui n'est autre qu'une histoire des rythmes en général, principalement à l'époque médiévale. Une telle étude fait défaut et j'espère combler prochainement cette lacune en montrant pour commencer comment la notion de rythme qui est la nôtre aujourd'hui est beaucoup plus large que celle qui prévalait dans l'Antiquité et au Moyen Âge, mais aussi comment cette notion moderne peut nous aider à mieux comprendre la «rythmicité» propre de la société et de la culture médiévales dans tous ses aspects. Ma réflexion porte ainsi sur les conceptions et les pratiques de la nature et du corps comme sur celles du temps et de la liturgie; sur la scansion des récits historiques comme sur les processions et le voyage; sur les

9. Laura Weigert, Weaving Sacred Stories: French Choir Tapestries and the Performance of Clerical Identity, Ithaca, Cornell University Press, 2004. Laura Weigert, Histoire de Saint-Étienne: la tenture du choeur de la cathédrale d'Auxerre, Auxerre, Musée d'art et d'histoire, 2000.

10. Le livre très suggestif de Kai Christian Ghattas, Rhythmus der Bilder. Narrative Strategien in Text- und Bildzeugnisen des 11. bis 13. Jahrhunderts, Cologne, Weimar et Vienne, Böhlau Verlag, 2009, compare les procédés narratifs de la Tapisserie de Bayeux à ceux de la Bible moralisée et à ceux de la littérature germanique contemporaine. La bibliographie concernant la Tapisserie de Bayeux est considérable. Outre les deux volumes de la collection Zodiaque: Lucien Musset, La tapisserie de Bayeux: œuvre d'art et document historique, La Pierre-qui-Vire, Zodiaque, 1989, puis Simone Bertrand, La tapisserie de Bayeux et la manière de vivre au $11^{e}$ siècle, La Pierre-qui-Vire, Zodiaque, 1966, on se reportera utilement à: Pierre Bouet, Brian Levy et François Neveux (dir.), La tapisserie de Bayeux: l'art de broder l'histoire, Actes du colloque de Cerisy-la-Salle (1999), Caen, Presses Universitaires de Caen, 2004. Pour la dimension idéologique de l'œuvre, voir l'important ouvrage de Suzanne Lewis, The Rhetoric of Power in the Bayeux Tapestry, Cambridge (Mass.) et New York, Cambridge University Press, 1999. Pour la comparaison avec le cinéma: Michel Parisse, La tapisserie de Bayeux. Un documentaire du $11^{e}$ siècle, Paris, Denoël, 1983. Je ne suis pas convaincu, pour ce qui est de l'origine de l'œuvre, par les hypothèses récentes de George Beech, Was the Bayeux Tapestry made in France? The Case for Saint-Florent of Saumur, New York, Palgrave Macmillan, 2005. 
«rythmes scolaires» (comme nous le dirions aujourd'hui) comme sur ceux de la fiscalité et du pouvoir. La Tapisserie de Bayeux a sa place à plus d'un titre dans cette réflexion d'ensemble: en tant qu'elle est une image dynamique qui soulève, pour l'historien de l'art ou celui des images, une multitude de questions, largement inspirées, comme je l'ai dit plus haut, par notre expérience de l'art contemporain; en tant qu'elle figure une histoire, et renvoie par cela même à toutes les conceptions de l'histoire et du récit historique en usage au Moyen Âge; en tant également qu'elle est un objet «total », à la fois ornemental, figuratif et linguistique, qui invite à rechercher des liens entre ces divers niveaux de l'expression du rythme: c'est du reste cette mise en relation qui représente, je pense, l'aspect le plus original et personnel de mon étude, aussi rapide soit-elle.

On admet aujourd'hui que cette broderie exceptionnelle fut réalisée une quinzaine d'années seulement après la victoire de Hastings, remportée le 14 octobre 1066 par le duc de Normandie Guillaume sur son rival Harold. Cette victoire permit à Guillaume de succéder sur le trône d'Angleterre au roi anglo-saxon Édouard et de jeter ainsi les bases du royaume anglo-normand. Plus précisément, la broderie a pu être réalisée entre 1077 (consécration de la cathédrale de Bayeux le 14 juillet 1077, en présence du nouveau roi Guillaume et de la reine Mathilde) et 1087 (mort de Guillaume) ou même seulement 1082 (année de la disgrâce de son frère Odon, destinataire probable de la broderie). Diverses analyses assurent que la broderie a été réalisée en Angleterre, dans le Kent, pour Odon, comte du Kent, évêque de Bayeux, demi-frère du duc Guillaume, qui avait participé, les armes à la main, à l'expédition ${ }^{11}$.

Rappelons pour mémoire que l'œuvre n’est pas une tapisserie (en dépit du nom qu'on lui donne communément), mais une broderie sur lin en fils de laine de huit couleurs et utilisant deux types de points (le point de tige et le point de couchage). L'ensemble se compose de huit lés de taille inégale (de 6,60 m à 13,75 m) cousus de bout en bout. En 1724, la broderie a été placée sur une toile sur laquelle les épisodes successifs ont été numérotés de 1 à 58 . Cette numérotation commode est utilisée par tous les commentateurs de l'œuvre, et je me tiendrai moi aussi à cet usage.

Contrairement à une idée reçue, cette œuvre ne dépeint pas seulement la conquête de l'Angleterre. La moitié des épisodes (1 à 25) concernent la mainmise du duc de Normandie sur la Bretagne en 1064. Après quoi, l'année 1065 est complètement occultée jusqu'au mois de décembre. Puis, la consécration de

11. Thomas Alexander Heslop, «Regarding the Spectators of the Bayeux Tapestry: Bishop Odo and his Circle», Art History, vol. 32, n² 2, avril 2009, p. 223-249. 
Saint-Pierre de Westminster (28 décembre 1065), la mort du roi Édouard et ses funérailles (le 5 janvier suivant), le couronnement de Harold (le 6 janvier), inaugurent une nouvelle série d'événements qui occupent l'année 1066: l'apparition de la comète de Halley entre le 24 avril et le $1^{\text {er }}$ mai, la traversée de la Manche du 27 au 28 septembre et la victoire de Hastings le 14 octobre 1066 (scènes 29 à 34).

La broderie a été maintes fois comparée, pour les informations qu'elle donne, aux autres sources, très nombreuses, dont nous disposons sur la conquête de l'Angleterre: le poème de Baudri de Bourgueil sur les tapisseries de la chambre d'Adèle de Blois et les chroniques contemporaines ou légèrement postérieures (Guillaume de Poitiers, Eadmer, Guillaume de Jumièges, Wace et d'autres). Mais dans aucune de ces œuvres, la relation des événements ne coïncide exactement avec la version en images qu'en donne la broderie ${ }^{12}$. Du reste, Lucien Musset a émis l'hypothèse que ce n'est pas la conquête, mais plutôt le parjure de Harold qui serait l'événement central de la broderie. Cette explication offre l'avantage de donner à celle-ci, souvent présentée comme la première grande œuvre artistique profane du Moyen Âge, une signification en rapport avec le sacré plus conforme à la nature habituelle des images médiévales. Cette hypothèse est confortée par la plus ancienne mention connue de la broderie, dans l'inventaire de 1476 de la cathédrale de Bayeux, qui précise qu'elle était exposée dans le chœur de cette église le jour anniversaire de la dédicace, lors de l'ostension des reliques conservées dans le sanctuaire. C'est précisément sur ces reliques que Harold a prêté serment, comme le montre la scène centrale de la broderie (scène 23).

On distingue dans la broderie trois éléments fondamentaux dont il s’agit de comprendre les relations:

- le récit en images de la bande centrale ( $33 \mathrm{~cm}$ de haut en moyenne, $70,34 \mathrm{~m}$ de long, mais il doit manquer à la fin 1,40 m environ, correspondant vraisemblablement à un dernier épisode); le récit est continu, mais il présente des étapes successives et une scansion dont on verra les modes d'expression. Ce récit se développe au gré des relations entre les trois personnages principaux: le roi Édouard d'Angleterre, son successeur Harold et son rival victorieux, le duc Guillaume de Normandie;

12. Lewis Thorpe, The Bayeux Tapestry and the Norman Invasion, with an Introduction and a Translation from the Contemporary Account of William of Poitiers, Londres, The Folio Society, 1973. 
- les inscriptions latines qui désignent au fil du récit les personnages et les actions représentés dans la bande centrale de la broderie;

- les bordures supérieure et inférieure de la broderie (7 cm de haut chacune). C'est par elles que je commence.

I. Les bordures consistent le plus souvent en une succession irrégulière de bâtons obliques et colorés, simples ou doubles, qui :

- tantôt forment des chevrons encadrant des couples d'animaux (quadrupèdes, oiseaux, hybrides) et des motifs végétaux répétitifs (fleurettes situées alternativement à la base ou au sommet de la bordure, ou rinceaux se déployant entre deux bâtonnets); de façon schématique, on peut représenter cette alternance irrégulière des chevrons par le dessin suivant: /.1./.1.//.11./I.11./.1./.।

- tantôt sont parallèles, irrégulièrement regroupés, mais toujours inclinés dans le sens du récit:

1.1.1.1.1./1./1./1.1.1.1././1./1/.

(Je représente par un simple point la figure végétale ou animale qu’encadrent les chevrons ou les bâtonnets parallèles.)

La première disposition est surtout présente dans la première moitié de la broderie, la seconde tend à se généraliser ensuite, quand se précipite l'action au moment de la bataille de Hastings. Cependant, les bordures ne forment pas un système clos, elles entrent en relation avec la bande narrative centrale.

D'une part, les bordures représentent en de nombreux endroits des microrécits, qui sont des fables animalières, regroupées surtout au début dans la bande inférieure, et qui semblent donner une sorte de fondement moral et didactique au récit principal, en préfigurant le parjure de Harold par des récits de tromperie, de violence et de châtiment connus de tous: le corbeau et le renard (scènes 4 , 16, 24); le loup et l'agneau, accusé de troubler l'eau de la rivière (scène 4); la louve qui ne veut pas rendre la tanière qui lui a été prêtée pour abriter ses petits (scène 4); le loup et la grue, qui accepte imprudemment de sortir un os coincé dans la gorge du carnassier (scènes 4-5 et 24); le roi loup qui enfreint son serment de ne pas dévorer les autres animaux (scène 5); le milan qui enlève la grenouille attachée par la patte à une souris (scène 5); le loup qui refuse, contrairement à son serment, de partager sa proie avec le bœuf, le mouton et le chien (scène 7); l'hirondelle qui incite les oiseaux à se méfier du paysan qui sème le lin dont il fera une fronde (scène 10). Quant à la fable du loup berné par la chèvre qu'il s'apprêtait à dévorer (scènes 7 et 51), elle montre, comme l'histoire de Harold 
et Guillaume, que la fourberie, loin de triompher, peut se retourner contre le trompeur ${ }^{13}$.

D'autre part, il arrive que la bande centrale déborde dans les bordures: soit dans la bordure supérieure, comme c'est le cas lors de la traversée de la baie du Mont-Saint-Michel (scènes 5-6); soit dans la bordure inférieure, envahie durant la bataille de Hastings par les morts mutilés et dépouillés de leur armure et plus loin par une rangée de petits archers en pleine action. Non seulement les corps brisés et mutilés prennent la place de la succession des bâtonnets dont on a parlé, mais ils en évoquent la disposition et la scansion, fondées sur la répétition et la variation des mêmes éléments visuels.

Il n'en reste pas moins que les bordures, même quand elles présentent un contenu figuratif, constituent avant tout un dispositif ornemental dynamique qui scande le déroulement du récit dans la bande centrale. Tout particulièrement, les bâtonnets colorés inclinés dans le sens du récit résonnent véritablement avec les actions figurées sur la broderie. Pour nous, elles évoquent par leur forme le battement d'une mesure, même si nous savons bien que la musique ignorait tout au $11^{\mathrm{e}}$ siècle de tels procédés graphiques, qui ne font leur apparition qu'avec la musica mensurabilis et la polyphonie vers 1200 . Il n'en est pas moins légitime de penser ici à une sorte de musique de la broderie, d'autant mieux que les inscriptions portées par celle-ci appelaient une lecture à haute voix et un commentaire public, donc une vocalisation puissante qui s'apparentait certainement à un chant.

II. La bande centrale déploie son rythme figuratif propre. Le récit est continu, mais la distinction entre les scènes est assurée par divers procédés: des arbres ou des bouquets d'arbres les délimitent (scènes 8, 9-10, 13-14), comme le font aussi les architectures des villes et des châteaux. Des éclaireurs, témoins ou espions observent les scènes, montrent les événements de la main, informent leur maître; ils donnent le sens de l'action aux autres personnages, mais le communiquent aussi au spectateur de la broderie: ainsi, dans la scène 4, lors du banquet à la cour d'Édouard, un homme debout au sommet de l'escalier regarde la scène vers la gauche tout en montrant du doigt à droite le départ de Harold vers la Normandie. Dans la scène 9, Harold et Guy «parlent», espionnés par un Normand qui se cache derrière une colonne, avant, dans la scène suivante, de venir informer Guillaume. Dans la scène 14, Guillaume ramène Harold dans

13. Léon Hermann, Les fables antiques de la broderie de Bayeux, Bruxelles et Berchem, Latomus, 1964. 


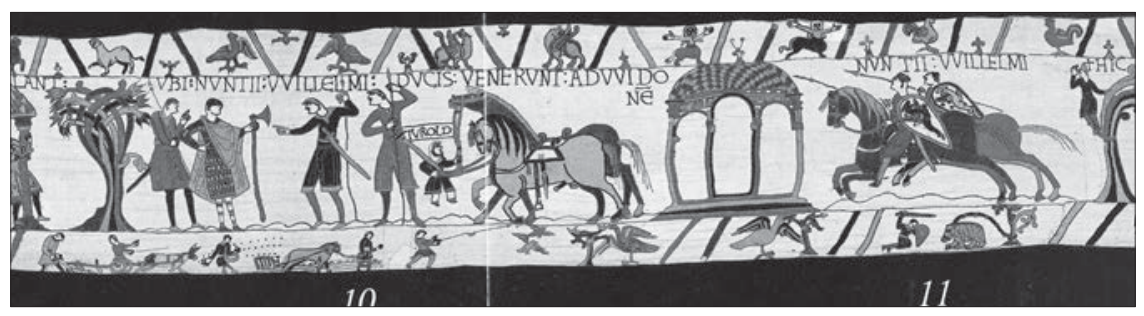

Fig. 1: Broderie, scènes 10-11. Les messagers du duc chevauchent de droite à gauche (Ubi nuntii Willemni ducis venereunt). Bordure : la fable du paysan qui sème du lin pour faire une fronde afin de chasser les oiseaux. Droits réservés, GAHOM.

son palais et un personnage devant la tour montre du doigt les arrivants. Dans la scène 24, Harold retourne en Angleterre, observé par un veilleur monté sur une tour. Dans les scènes 31-32, un groupe de cinq hommes observent vers la gauche le couronnement de Harold, tandis qu'un autre groupe de cinq hommes - les mêmes peut-être -, précédés par un sixième individu, montrent du doigt l'apparition de la comète. Dans la scène 50 , au sommet d'un monticule, un soldat anglais renseigne Harold sur les positions des Normands: il est tourné vers la gauche, puis vers la droite, et sa lance se croise avec elle-même, ce qui renforce l'impression de la succession immédiate et de la relation de cause à effet entre ces deux actions.

Le récit se déroule de gauche à droite, mais l'action s'inverse à deux moments importants au moins. Premièrement, quand Guillaume, averti de la capture de Harold par Gui de Ponthieu, envoie des messagers à celui-ci pour qu'il lui amène le captif (scènes 12, 11, 10) (Fig. 1). La broderie représente successivement 1) la capture de Harold, 2) les messagers du duc qui, en chevauchant de la droite vers la gauche - à rebours de l'orientation générale du récit - viennent donner à Gui de Ponthieu l'ordre de livrer le captif au duc Guillaume, 3) le duc Guillaume informé par un espion de la capture de Harold (ce qui motive l'envoi des messagers), et enfin 4) Gui de Ponthieu conduisant son prisonnier à Guillaume. On voit bien que cet ordre de succession des actions n'est pas l'ordre dans lequel les événements se sont effectivement déroulés: on devrait voir au contraire se succéder chronologiquement de gauche à droite 1), 3), 2), 4). Le récit en images s'enroule sur lui-même, ce qui peut répondre à un principe d'économie: en faisant jouer simultanément plusieurs rôles aux mêmes personnages, on diminue le nombre des figures à représenter; ainsi Gui de Ponthieu est-il figuré simultanément en tant qu'il arrête Harold et en tant qu'il reçoit l'ordre de le livrer à Guillaume. Mais surtout, l'enroulement du récit sur lui-même met en valeur la volonté du duc Guillaume (dont les messagers, chevauchant en sens inverse 


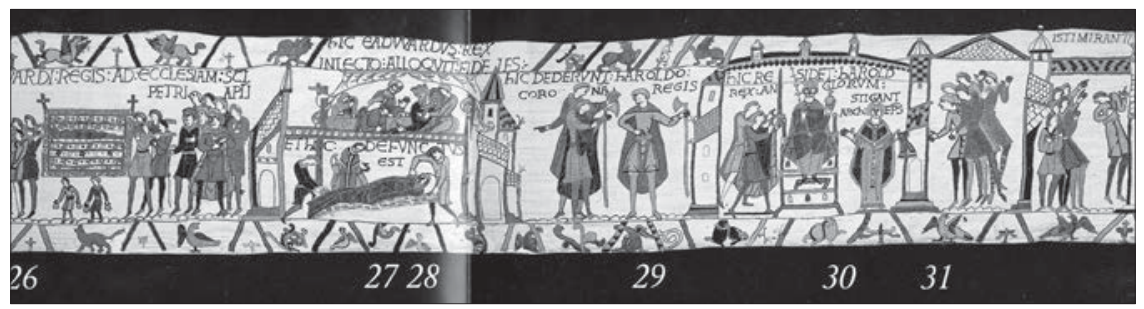

Fig. 2: Broderie, scènes 26-32. Les funérailles du roi Édouard à Westminster : de droite à gauche. Le couronnement de Harold (Hic portatur corpus Edwardi ad ecclesiam Petri Apostoli. Hic Edwardus rex in lecto alloquitur fideles et hic defunctus est. Hic dederunt Haroldo coronam regis). Droits réservés, GAHOM.

du déroulement général de la broderie, transmettent l'ordre de livrer le captif), donc le fait que toute l'action dépend de lui, que rien ni personne - ni vassal, ni rival - ne peut lui résister.

Cette volonté de valoriser un personnage ou un lieu apparaît aussi comme la raison d'être de la seconde inversion du même type, aux scènes 26, 27 et 28, où les funérailles du roi Édouard à Westminster précèdent la double scène de sa mort, avant que le récit ne reprenne son sens habituel avec le couronnement de Harold (Fig. 2). Ici, ce qui est privilégié est l'image centrale de l'abbaye de Westminster, dont l'église fut consacrée le 28 décembre 1065, juste à temps pour accueillir la dépouille mortelle du roi Édouard, décédé le 5 janvier. L’ordre chronologique 1) consécration de l'église, 2) mort du roi Édouard, 3) funérailles du roi, 4) couronnement de Harold, s'inverse dans le déroulement de la broderie au profit de la séquence 1), 3), 2), 4). L'abbaye est le point central vers lequel converge la procession funéraire, à rebours du déroulement du récit. Il est probable que l'église abbatiale figurait de nouveau à la fin, malheureusement perdue, de la broderie, où devait être représenté le couronnement à Westminster de Guillaume comme roi d’Angleterre et légitime successeur du roi Édouard.

Lallure et la vitesse des chevaux, qui alternativement marchent au pas (scènes 48-49), se mettent au trot, s'élancent au galop, puis reviennent à une allure modérée ou s'arrêtent (scène 50) confèrent au récit son dynamisme rythmique le plus puissant (Fig. 3). Celui-ci est encore renforcé par l'effet de masse des troupes de cavaliers, qui tantôt s'étirent en longueur et tantôt se regroupent sur plusieurs plans, tandis que les silhouettes de couleurs différentes (rouge, noir, ocre, et parfois gris ou brun), leur recouvrement partiel grâce à la superposition des plans, la démultiplication des pattes des chevaux, ajoutent à l'effet de mouvement. Le galop des chevaux fait inévitablement penser aux expériences chronophotographiques d'Étienne-Jules Marey évoquées au début de cet article. Du reste, c'est notre familiarité avec les techniques du cinéma 


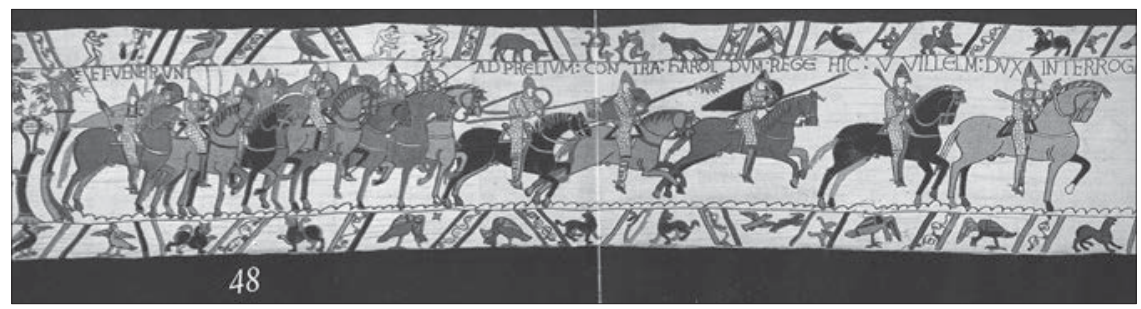

Fig. 3: Broderie, scènes 48-49. Les cavaliers passent du pas au galop (Et venerunt ad prelium contra Haroldum rege (m). Hic William dux interrogat). Droits réservés, GAHOM.

qui nous rend sensibles à la valeur dynamique de la Tapisserie de Bayeux et au caractère exceptionnel de cet objet. Certes, on doit reconnaître que d'autres civilisations contemporaines de notre Moyen Âge (mais sans aucun contact avec lui) ont su développer à une bien plus grande échelle encore ce type de figuration: je pense par exemple aux frises en bas relief, figurant semblablement des scènes de combat, des temples khmers d'Angkor Vat ou du Bayon $\left(12^{\mathrm{e}}-13^{\mathrm{e}}\right.$ siècles). Mais les artisans qui ont réalisé la broderie de Bayeux n’avaient pas une moindre capacité, véritablement stupéfiante, d'observation et d'expression du mouvement. L'étroitesse de la bande centrale, tout entière occupée en hauteur par les silhouettes des chevaux, canalise leur course et accroît la puissance d'entraînement de leur chevauchée. La même préoccupation rythmique se retrouve dans les files de navires de la flotte normande (scène 36 et suivantes) et dans la superposition des boucliers accrochés aux bat-flancs ou portés par les cavaliers dans la bataille.

III. La troisième dimension à prendre en compte est linguistique : les inscriptions qui surmontent les scènes et s'introduisent entre les personnages ont pour fonction, soit d'identifier les personnages (Edwardus rex, «Le roi Édouard»; Nuntii Wilelmi, «Les messagers de Guillaume»; Harold) et des lieux (Ecclesia, «L'église»), soit d'attirer le regard du spectateur sur l'action en cours et de lui permettre d'identifier les scènes: il s'agit le plus souvent de phrases complètes introduites par les adverbes de lieu Hic, «Ici» (par exemple: Hic venit nuntius ad Wilgelmum ducem, «Ici le messager vient/vint auprès du duc Guillaume ») ou plus rarement Ubi, «Là » (Ubi Harold (et) Wido parabolant, «Là, Harold et Gui parlent ensemble») ou par un démonstratif (Isti mirant stellam, «Ceux-ci observent l'étoile»). Dans bien des cas, la conjonction «et» fait rebondir la phrase pour décrire deux ou même trois actions consécutives (Hic apprehendit Wido Haroldum et duxit eum ad Belrem et ibi eum tenuit, «Ici, Gui capture Harold 
et le conduisit à Belrem et là le tient prisonnier ») : cette structure cumulative est très fréquente puisqu'on la repère 14 fois, tandis que les phrases simples sont au nombre de 27. L'alternance de temps verbaux contribue également à rythmer le «phrasé» de la broderie: outre les temps indéterminés (ainsi l'un des verbes les plus fréquents, venit, peut être un présent («il vient») ou un parfait («il vint»), à l'inverse du pluriel venerunt, «ils vinrent», dont le temps du passé est indubitable), on trouve tantôt des verbes au présent (interrogat, nuntiat, parabolant, «il interroge», «il annonce», «ils parlent», etc.), tantôt des verbes au parfait (duxit, exierunt, «il conduisit», «ils sortirent», etc.) ou exceptionnellement à l'imparfait (Hic Haroldus trahebat de arena, "Ici, Harold tirait hors du sable»). Les verbes au passé sont plutôt plus nombreux dans la première moitié de la broderie, les verbes au présent se multiplient en revanche au moment de la bataille de Hastings: on peut donc penser que l'usage de plus en plus fréquent du temps présent vise à produire un effet de "présentification ", à rendre effectivement présents aux spectateurs les combats et la victoire de Guillaume. Mais ce qui frappe est plutôt l'alternance des temps et le passage d'un temps à l'autre, jusque dans une même phrase, soit qu'un présent cède la place à un passé (Hic milites Willelmi ducis pugnant contra Dinantes et Cunan claves porrexit, «Ici, les soldats du duc Guillaume combattent ceux de Dinant et Conan tendit les clefs »; Hic exeunt caballi de navibus et hic milites festinaverunt Hestinga ut cibum raperentur, «Ici, les chevaux sortent des navires et ici les soldats se hâtèrent en direction de Hastings pour s'y emparer de la nourriture »; Hic coquitur caro et hic ministraverunt ministri, «Ici on cuit la viande et ici les serviteurs vaquèrent à leur service»; Hic Franci pugnant et ceciderunt qui erant cum Haroldo, «Ici les Français combattent et furent tués ceux qui étaient avec Harold»), soit, inversement, qu'un passé soit suivi d'un présent (Hic fecerunt prandium et hic episcopus cibum et potum benedicit, "Ici ils prirent un repas et ici l'évêque bénit la nourriture et la boisson»). C'est cette alternance, le balancement entre des formes verbales diverses, qui contribue à donner sa «musique» vocale à la broderie.

Ce type d'alternance des temps grammaticaux présent et passé n'est pas unique: on l'observe aussi, mais dans un rapport de proportions inverses, dans la Chanson de Roland ${ }^{14}$. Mais la comparaison est difficile parce que la valeur du passé et du présent n’est pas nécessairement la même en ancien français et en latin. Du moins les deux œuvres ont-elles en commun de constituer une

14. George Beech, «The Alternation Between Present and Past Time in the Telling of the Bayeux Tapestry Story ", Annales de Normandie, vol. 58, ${ }^{\circ} 1$ et 2, juin 2008, p. 7-23. 
mémoire historique, en rendant présents et en confiant à la mémoire des hommes des événements glorieux qui appartiennent à un passé plus ou moins récent.

On peut encore affiner l'analyse : la première partie de la broderie, qui porte sur les événements antérieurs à 1066, fait un usage massif du passé (20 verbes au passé, 3 seulement au présent). À partir de la mort d'Édouard et du couronnement de Harold, le rapport s'inverse (26 verbes au présent, 16 au passé) : désormais le spectateur est invité à participer « en direct» à l'action, comme si elle se déroulait devant lui.

Qu'il s'agisse des bordures, des images de la bande centrale ou des légendes qui les font parler en invitant à un commentaire de vive voix, on retiendra ainsi que la broderie de Bayeux utilise pleinement toutes les caractéristiques de ce que nous nommons le rythme: d'abord la répétition, par exemple celle des bâtonnets parallèles ou des chevrons qui animent les bandes ornementales supérieure et inférieure; mais aussi les figures des chevaux et des soldats, tous représentés suivant le même modèle. Deuxièmement et tout en même temps, la variété: la «varietas» est une valeur très importante dans la culture et les textes du Moyen Âge, où elle peut être prise en mauvaise part, quand ce qui est «divers » trouble l'ordre des hommes et de Dieu; mais ici, elle est une valeur positive: la «variété » multiplie les écarts dans la répétition et crée ainsi le mouvement dans les images, la dynamique des scènes, par exemple dans l'alternance des couleurs selon plusieurs combinaisons possibles, et aussi celle des formes (comme les pattes des chevaux ou les boucliers des fantassins et des cavaliers). La broderie ne présente pas un flux narratif régulier et continu: elle connaît des temps d'arrêt, des reprises, des accélérations (particulièrement nettes dans la bataille); tantôt elle s'efforce de lier les actions successives, tantôt, au contraire, elle les juxtapose; en principe, l’ordre des scènes figurées et l'ordre chronologique du récit ne font qu'un, mais il y a des omissions (presque toute l'année 1065 manque) et surtout la broderie fait plusieurs fois retour sur elle-même, montrant par exemple les funérailles du roi Édouard avant sa mort et ce à contre-courant (de droite à gauche) du sens habituel du récit (de gauche à droite), redoublé par le sens de l'écriture des inscriptions. À propos de ces dernières, l'alternance des temps verbaux et la prépondérance croissante du temps présent doivent être interprétées en relation avec l'ensemble des autres éléments figurés et chromatiques de la broderie, car ils participent de la nature rythmique de l'objet comme de sa réception visuelle et verbale, lors de la commémoration liturgique annuelle de la dédicace de l'église cathédrale de Bayeux, lors de l'ostension solennelle des reliques sur lesquelles Harold avait prêté serment. 